\title{
An Improved 'Heuristic' Approximation for the Period of a Nonlinear Pendulum: Linear Analysis of a Classical Nonlinear Problem
}

\author{
A. Beléndez, A. Hernández, T. Beléndez, A. Márquez and C. Neipp \\ Departamento de Física, Ingeniería de Sistemas y Teoría de la Señal. \\ Universidad de Alicante. Apartado 99. E-03080 Alicante. SPAIN \\ E-mail: a.belendez@ua.es,Fax:+34-96-5909750
}

\begin{abstract}
A new analytical approximate expression for the period of the simple pendulum is obtained by using a heuristic but pedagogical derivation. This formula depends on two parameters obtained by comparing term-by-term the power-series expansions of the approximate and exact expressions for the period. This formula is compared with others in the literature and the numerical results obtained show that the published approximations are not nearly as good as the new expression proposed in this paper.
\end{abstract}

Keywords: Nonlinear oscillator; Simple pendulum; Large-angle period; Approximate solutions.

\section{Introduction}

Considerable attention has been directed towards the study of nonlinear problems in all areas of physics and engineering [1]. It is very difficult to solve nonlinear problems and, in general, it is often more difficult to get an analytic approximation than a numerical one for a given nonlinear problem. In particular the study of nonlinear oscillators is of great interest to many researchers $[2,3]$. There are several methods used to find approximate solutions to nonlinear oscillators, such as perturbation techniques [4-14] or harmonic balance based methods [15-22]. Surveys of the literature with numerous references and useful bibliographies have given in $[3,23]$. In general, given the nature of a nonlinear phenomenon, the approximate methods can only be applied within certain ranges of the physical parameters and to certain classes of problems [11].

In physics and engineering there are some simple systems for which the equations governing their behaviour are easy to formulate but whose mathematical resolution is complicated. The mathematical modeling of these systems often results in non-linear differential equations that in some cases can be linearized by replacing the non-linear equation by a linear equation that approximates the original non-linear equation closely enough to give useful results [2]. There are innumerable oscillatory systems that can be modelled as second order, nonlinear, ordinary differential equations. One of the simplest and more famous nonlinear oscillating systems is the free pendulum. Application of Newton's second law to this physical system gives a differential equation with a non-linear term (the sine of an angle). It is possible to find the integral expression for the period of the pendulum and to express it in terms of elliptic functions. However, if we consider the case of small oscillations it is possible to substitute the sine of the angle by the angle itself, thereby obtaining a second order linear differential equation with constant coefficients, the solution of which is the well-known harmonic function, and an amplitude-free period is obtained.

Over the years several approximation schemes have been developed to investigate the situation for large amplitude oscillations of a simple pendulum, and in this paper we provide yet another. The approximate expression for the period of the simple pendulum derived here is quite accurate for very large amplitudes and we 
will see that this expression is more accurate than other similar, simple approximations suggested by other authors [24-26].

The approximate expression proposed in this paper depends on two parameters which are found by comparing term-by-term the powerseries expansions of the approximate and exact expressions for the period of the pendulum. This implies that the expansion of the proposed formula to the third term is the same as the expansion of the exact solution.

Other approximations analyzed in this paper and suggested by other authors [24-26] have only one parameter and comparing their powerseries expansions with the exact one, the series coincide only up to the second term. We use these last approximations as the starting point for improvement of the formulas, and the quality of the expression obtained is better than that of the original ones.

\section{Some approximate expressions for the period of a free pendulum}

The differential equation modeling the free, undamped simple pendulum is

$$
\frac{\mathrm{d}^{2} \theta}{\mathrm{dt}^{2}}+\frac{g}{l} \sin \theta=0
$$

where $\theta$ is the angular displacement, $l$ is the length of the pendulum, $t$ is the time and $g$ is the acceleration due to gravity. On the other hand $\theta_{0}$ will be the amplitude of oscillations. Equation (1), although straightforward in appearance, is in fact rather difficult to solve because of the nonlinearity of the trigonometric function $\sin \theta$. There are no analytical solutions for the above differential equation. In fact, the solution is expressed in terms of elliptic integrals [27, 28].

There are various linearized approximations for the non-linear equation (1). In the simplest of these, the analysis is limited to the case of small amplitudes (small values for $\theta_{0}$ ). Note that if $\theta$ is small, then the sine function can be approximated by $\sin \theta \approx \theta$, and the non-linear differential equation (1) becomes a linear differential equation that can easily be solved, and the period $T_{0}$ of the oscillation is given by

$$
T_{0}=2 \pi \sqrt{\frac{l}{g}}
$$

The period for this case is independent of the amplitude $\theta_{0}$ of oscillations and is only a function of the length $l$ of the pendulum and the acceleration of gravity $g$.

In other linearized approximations the $\sin \theta$ term is substituted in equation (1), not as in the previous case by the linear function $\theta$, but by a function proportional to the angle $\theta$, with the proporcionality 'constant' being a function $f\left(\theta_{0}\right)$ of the amplitude $\theta_{0}$

$$
\sin \theta \approx f\left(\theta_{0}\right) \theta
$$

where $f\left(\theta_{0}\right)$ must be determined.

Substituting equation (3) into equation (1) gives

$$
\frac{\mathrm{d}^{2} \theta}{\mathrm{dt}^{2}}+\frac{g}{l} f\left(\theta_{0}\right) \theta=0
$$

which is a linear differential equation easily integrable, and the approximate period of the oscillation $T_{\text {app }}$ is given by

$$
\frac{T_{\text {app }}}{T_{0}}=\frac{1}{\sqrt{f\left(\theta_{0}\right)}}
$$

where $T_{0}$ is the period for small amplitude oscillations and is given by equation (2). As can be seen in equation (5), the period $T_{\text {app }}$ depends on the amplitude $\theta_{0}$.

Once this linear approximation has been made the problem is to find the optimum expression for the function $f\left(\theta_{0}\right)$, which is done by comparing the exact value $T_{\text {ex }}$ of the period of a simple pendulum with the approximate value in equation (5). This is done by making a termby-term comparison of the series expansion on the right-hand side of equation (5) with the corresponding series of $T_{\text {ex }}$, which implies taking into account the series expansion for the complete elliptic integral of the first kind. In any case it is necessary to have an expression for the function $f\left(\theta_{0}\right)$ which includes a parameter whose optimum value may be calculated comparing the series expansions mentioned above. 
One simple possibility, suggested by Molina [25], is

$$
f\left(\theta_{0}\right)=\left(\frac{\sin \theta_{0}}{\theta_{0}}\right)^{n}
$$

where $n$ is a positive constant to be determined. In another paper Ganley [24] (and more recently Parwani[26]) suggested the expression

$$
f\left(\theta_{0}\right)=\left(\frac{\sin m \theta_{0}}{m \theta_{0}}\right)
$$

where $m$ is also a positive constant to be determined. In both equation (6) and equation (7) there is a parameter to be determined that is obtained by comparing the first terms of the power-series expansion for equations (6) and (7) with the corresponding first terms of the powerseries expansion for the exact period [12]

$$
\frac{T_{\mathrm{ex}}}{T_{0}} \approx 1+\frac{1}{16} \theta_{0}^{2}
$$

so that $n=3 / 4$ is obtained with Molina's approximation and $m=\sqrt{3} / 2$ with Ganley's.

Thus, the expressions for the approximate periods are

$$
\frac{T_{\text {app } 1}}{T_{0}}=\left(\frac{\sin \theta_{0}}{\theta_{0}}\right)^{-3 / 8}
$$

with Molina's approximation, and

$$
\frac{T_{\text {app2 }}}{T_{0}}=\left[\frac{\sin \left(\sqrt{3} \theta_{0} / 2\right)}{\sqrt{3} \theta_{0} / 2}\right]^{-1 / 2}
$$

with Ganley's.

The quality of these approximations can be determined by comparing them to the exact value of the period of oscillations that is given by the equation $[2,3]$

$$
\frac{T_{\mathrm{ex}}}{T_{0}}=\frac{2}{\pi} K(q)
$$

where $q=\sin ^{2}\left(\theta_{0} / 2\right)$ and $K(q)$ is the complete elliptic integral of the first kind. However, instead of doing this, first we can compare termby-term the power-series expansion of the exact period of the pendulum

$$
\frac{T_{\mathrm{ex}}}{T_{0}} \approx 1+\frac{1}{16} \theta_{0}^{2}+\frac{11}{3072} \theta_{0}^{4}+\frac{173}{737280} \theta_{0}^{6}+\ldots
$$

with the corresponding power-series expansions of the approximate expressions for the period of the pendulum (from equation (9))

$$
\frac{T_{\text {app } 1}}{T_{0}} \approx 1+\frac{1}{16} \theta_{0}^{2}+\frac{12.4}{3072} \theta_{0}^{4}+\frac{223.5}{737280} \theta_{0}^{6}+\ldots
$$

and (from equation (10))

$\frac{T_{\text {app } 2}}{T_{0}} \approx 1+\frac{1}{16} \theta_{0}^{2}+\frac{10.8}{3072} \theta_{0}^{4}+\frac{156.9}{737280} \theta_{0}^{6}+\ldots$

If we compare term-by-term the exact equation (12) with the approximate equations (13) and (14) we can see that the two latter equations coincide with the exact equation only up to the second term $\left(\theta_{0}^{2}\right)$, and the other terms are different. Since the first two terms of these series are identical, series (13) and (14) track series (12) closely for small amplitudes, but as the coefficients of the third (and subsequent) terms of series (13) and (14) are different to their counterparts in series (12), the difference between these approximate expressions for the period of the pendulum and the exact value increases as the amplitude of the oscillation increases. Even so, and as shown in the reference [26], equation (10) is a better approximation to the exact value than is equation (9), as can be seen by comparing the coefficients of the powers $\theta_{0}^{4}$ y $\theta_{0}^{6}$ of the series expansions of the exact and approximate periods.

\section{A more accurate approximation}

We shall now see that is it possible to find a linearized approximation of equation (3) that is better than those of equations (6) and (7). As has been shown, in both equation (6) and equation (7) there is only one unknown positive constant, 
so when we make a comparison with the exact value, this can only be done by considering the series expansion of the exact period up to the power $\theta_{0}^{2}$. If we considered an approximate function $f\left(\theta_{0}\right)$ with two unknown parameters, it would be possible to compare the series expansion of the approximate period obtained with this function $f\left(\theta_{0}\right)$ with the series expansion of the exact period but in this case taking into consideration not only the coefficient of the power $\theta_{0}^{2}$ but also that of the power $\theta_{0}^{4}$. Taking Molina's (equation (6)) and Ganley's (equation (7)) approximations as out starting point, we suggest the following expression for function $f\left(\theta_{0}\right)$

$$
f\left(\theta_{0}\right)=\left(\frac{\sin m \theta_{0}}{m \theta_{0}}\right)^{n}
$$

thus the approximate period is given by

$$
\frac{T_{\text {app } 3}}{T_{0}}=\left(\frac{\sin m \theta_{0}}{m \theta_{0}}\right)^{-n / 2}
$$

Obviously $T_{\text {app3 }}=T_{\text {app } 1}$, when $n=3 / 4=0.75$ and $m=1$ (Molina [24]), whereas $T_{\text {app3 }}=T_{\text {app2, }}$, when $n=1$ and $m=\sqrt{3} / 2=0.8660$ (Ganley [25]). In order to obtain optimum values for the parameters $m$ and $n$ in equation (16), the powerseries expansion of the right-hand side of equation (16) is performed -for example, it is easy to do this by using one of the packages of commercial software available, such as MATHEMATICA - and we obtain

$$
\frac{T_{\text {app3 }}}{T_{0}} \approx 1+\frac{1}{12} m^{2} n \theta_{0}^{2}+\frac{4 n+5 n^{2}}{1440} m^{4} \theta_{0}^{4}+\ldots
$$

Comparing term by term the series expansions of equations (12) and (17) it can be seen that it is necessary to simultaneously satisfy the equations

$$
\begin{gathered}
\frac{1}{12} m^{2} n=\frac{1}{16} \\
\frac{4 n+5 n^{2}}{1440} m^{4}=\frac{11}{3072}
\end{gathered}
$$

which make up a system of equations with two unknowns that may be solved without too much difficulty. The solution obtained is $n=24 / 25=$ 0.96 and $m=5 \sqrt{2} / 8=0.8839$, and equation (16) becomes

$$
\frac{T_{\text {app } 3}}{T_{0}}=\left[\frac{\sin \left(5 \sqrt{2} \theta_{0} / 8\right)}{5 \sqrt{2} \theta_{0} / 8}\right]^{-12 / 25}
$$

Comparing the values obtained for $n$ and $m$ with those corresponding to the approximations of a single parameter, it can be seen that the new approximation proposed in this study is closer to Ganley's approximation than to Molina's. This is only logical since as shown in the previous section when compared with the series expansion of the exact period, Ganley's (or Parwani's) approximation is better than Molina's. Table 1 shows the values of $n$ and $m$ for the different approximations analyzed in this study.

Table 1. Values of parameters $n$ and $m$ for the different approximations analyzed in this paper.

\begin{tabular}{lcc}
\hline Approximation & $n$ & $m$ \\
\hline Molina & 0.75 & 1.0000 \\
\hline Ganley - Parwani & 1.00 & 0.8660 \\
\hline This paper & 0.96 & 0.8839 \\
\hline
\end{tabular}

In order to determine the quality of this new approximation we compare equation (12), that is, the series expansion of the exact period with the series expansion of equation (20) that we can write as follows

$$
\frac{T_{\mathrm{app} 3}}{T_{0}} \approx 1+\frac{1}{16} \theta_{0}^{2}+\frac{11}{3072} \theta_{0}^{4}+\frac{164.5}{737280} \theta_{0}^{6}+\ldots
$$

It can be seen that both coincide up to the term $\theta_{0}^{4}$ and furthermore the coefficient of the power $\theta_{0}^{6}$ is also closer to the expansion coefficient of the exact period $(164.5 / 737280$ versus $173 / 737280)$ than the corresponding coefficients using Molina's (223.5/737280) and Ganley's (156.9/737280) approximations. The values of 
four expressions $T_{\text {ex }} / T_{0}$ (equation (11)), $T_{\text {app } 1} / T_{0}$ (equation (9)), $\quad T_{\text {app } 2} / T_{0}$ (equation 10)) and $T_{\text {app } 3} / T_{0}$ (equation (20)) for different values of the amplitude $\theta_{0}$ are presented in Table 2, which shows that the new approximation $T_{\text {app3 }}$ introduces in this paper gives a greater accuracy than the other approximations. Thus for example, the relative error between the approximate values and the exact value is less than $1 \%$ for amplitudes $\theta_{0} \leq 143^{\circ}$ if we consider the approximation suggested in this paper.

Table 2. Values of the four expressions of the period as a function of the amplitude of oscillations

\begin{tabular}{ccccc}
\hline$\theta_{0}$ & $\begin{array}{c}T_{\text {ex }} / T_{0} \\
\text { exact }\end{array}$ & $\begin{array}{c}T_{\text {app1 }} / T_{0} \\
\text { Molina }\end{array}$ & $\begin{array}{c}T_{\text {app2 }} / T_{0} \\
\text { Ganley- } \\
\text { Parwani }\end{array}$ & $\begin{array}{c}T_{\text {app3 } 3} / T_{0} \\
\text { this paper }\end{array}$ \\
\hline $10^{\circ}$ & 1.0019 & 1.0019 & 1.0019 & 1.0019 \\
$30^{\circ}$ & 1.0174 & 1.0174 & 1.0174 & 1.0174 \\
$50^{\circ}$ & 1.0498 & 1.0501 & 1.0497 & 1.0498 \\
$70^{\circ}$ & 1.1021 & 1.1034 & 1.1019 & 1.1021 \\
$90^{\circ}$ & 1.1803 & 1.1845 & 1.1794 & 1.1800 \\
$110^{\circ}$ & 1.2953 & 1.3072 & 1.2922 & 1.2939 \\
$130^{\circ}$ & 1.4698 & 1.5026 & 1.4588 & 1.4636 \\
$150^{\circ}$ & 1.7622 & 1.8605 & 1.7192 & 1.7326 \\
$170^{\circ}$ & 2.4394 & 2.8990 & 2.1787 & 2.2240 \\
\hline
\end{tabular}

\section{Conclusions}

In this paper, three approximations for the period of a nonlinear pendulum are discussed. All of them are simple, but the one suggested by us is more accurate than the others proposed earlier by Molina [25] and Ganley [24]-Parwani [26]. The main result (20) is relatively simple and accurate for very large amplitude oscillations, and is better than other linearized approximations previously published. In fact, the percent error of this approximation remains less than $1 \%$ for angles up to $143^{\circ}$. However, it is important to point out that when one of these expressions is used to calculate the period of the pendulum, they are using an expression that is not exact but only a convenient approximation that allows them to easily obtain the value of the period with sufficient accuracy. Finally, this linear analysis of the nonlinear oscillations of a free pendulum can be easily extended to any other oscillators, and the present paper can be used as paradigm for many other applications in analyzing the period of various nonlinear oscillators.

\section{Acknowledgements}

This work was supported by the "Ministerio de Educación y Ciencia", Spain, under project FIS2005-05881-C02-02, and by the "Generalitat Valenciana", Spain, under project ACOMP/ 2007/020.

\section{References}

[1] D. K. Campbell, Nonlinear science: the next decade (MIT Press, Massachusetts 1992).

[2] R. E. Mickens, Oscillations in Planar Dynamics Systems (World Scientific, Singapore 1996).

[3] J. H. He, Non-perturbative methods for strongly nonlinear problems (dissertation.deVerlag im Internet GmbH, Berlin 2006).

[4] J. H. He, "A new perturbation technique which is also valid for large parameters", $J$. Sound Vib. 229, 1257-1263 (2000).

[5] J. H. He, "Modified Lindstedt-Poincare methods for some non-linear oscillations. Part III: double series expansion", Int. J. Nonlinear Sci. Numer. Simulation 2, 317-320 (2001).

[6] J. H. He, "Modified Lindstedt-Poincare methods for some non-linear oscillations. Part I: expansion of a constant", Int. J. Non-linear Mech. 37, 309-314 (2002).

[7] J. H. He, "Modified Lindstedt-Poincare methods for some non-linear oscillations. Part II: a new transformation", Int. J. Non-linear Mech. 37, 315-320 (2002).

[8] P. Amore and A. Aranda, "Improved Lindstedt-Poincaré method for the solution of nonlinear problems", J. Sound. Vib. 283, 1115-1136 (2005).

[9] P. Amore and F. M. Fernández, "Exact and approximate expressions for the period of anharmonic oscillators", Eur. J. Phys. 26, 589-601 (2005). 
[10] J. H. He, "Homotopy perturbation method for bifurcation on nonlinear problems", Int. J. Non-linear Sci. Numer. Simulation. 6, 207208 (2005).

[11] A. Beléndez, A. Hernández, T. Beléndez, E. Fernández, M. L. Álvarez and C. Neipp, "Application of He's homotopy perturbation method to the Duffing-Harmonic oscillator", Int. J. Non-linear Sci. Numer. Simulation 8, 79-88 (2007).

[12] A. Beléndez, A. Hernández, T. Beléndez, C. Neipp and A. Márquez, "Application of the homotopy perturbation method to the nonlinear pendulum", Eur. J. Phys. 28, 93104 (2007).

[13] D. H. Shou and J. H. He, "Application of parameter-expanding method to strongly nonlinear oscillators", Int. J. Non-linear Sci. Numer. Simulation 8, 121-124 (2007).

[14] H. Hu, "Solution of a Duffing-harmonic oscillator by an iteration procedure", J. Sound vib. 298, 446-452 (2006).

[15] R. E. Mickens, "Comments on the method of harmonic-balance", J. Sound. Vib. 94, 456460 (1984).

[16] R.E. Mickens, "Mathematical and numerical study of the Duffing-harmonic oscillator", $J$. Sound Vib. 244, 563-567 (2001).

[17] B. S. Wu and C. W. Lim, "Large amplitude nonlinear oscillations of a general conservative system", Int. J. Non-linear Mech. 39, 859-870 (2004).

[18] C. W. Lim and B. S. Wu, "Accurate higherorder approximations to frequencies of nonlinear oscillators with fractional powers", J. Sound Vib. 281, 1157-1162 (2005).
[19] A. Beléndez, A. Hernández, A. Márquez, T. Beléndez and C. Neipp, "Analytical approximations for the period of a simple pendulum", Eur. J. Phys. 27, 539-551 (2006).

[20] A. Beléndez, A. Hernández, T. Beléndez, M. L. Álvarez, S. Gallego, M. Ortuño and C. Neipp, "Application of the harmonic balance method to a nonlinear oscillator typified by a mass attached to a stretched wire", J. Sound Vib. 302, 1018-1029 (2007).

[21] H. Hu and J. H. Tang, "Solution of a Duffingharmonic oscillator by the method of harmonic balance", J. Sound Vib. 298, 637639 (2006).

[22] C. W. Lim and B. S. Wu, "A new analytical approach to the Duffing-harmonic oscillator", Phys. Lett.A 311, 365-373 (2003).

[23] J. H. He, "Some asymptotic methods for strongly nonlinear equations", Int. J. Mod. Phys. B, 20, 1141-1199 (2006).

[24] M. I. Molina, "Simple linearizations of the simple pendulum for any amplitude", Phys. Teach. 35, 489-490 (1997).

[25] W. P. Ganley, "Simple pendulum approximation”, Am. J. Phys. 53 73-76 (1985).

[26] R. R. Parwani, “An approximate expression for the large angle period of a simple pendulum," Eur. J. Phys. 25, 37-49 (2004).

[27] J. B. Marion, Classical Dynamics of Particles and Systems (Harcourt Brace Jovanovich, San Diego, 1970)

[27] L. M. Milne-Thomson, "Elliptic integrals" in M. Abramowitz and I. A. Stegun (Eds.), Handbook of Mathematical Functions (Dover Publications, Inc., New York, 1972). 